\title{
A Case Report of Chronic Expanding Hematoma Initially Misdiagnosed as a Soft Tissue Sarcoma
}

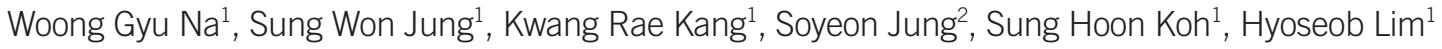 \\ ${ }^{1}$ Department of Plastic and Reconstructive Surgery, Hallym University Sacred Heart Hospital, Hallym University, Anyang, Korea; ${ }^{2}$ Department of Plastic and \\ Reconstructive Surgery, Chang Gung Memorial Hospital, College of Medicine, Chang Gung University, Taoyuan, Taiwan
}

\begin{abstract}
The differential diagnosis of a large soft tissue mass is challenging, and is further complicated by the difficulty of differentiating chronic expanding hematoma from a malignant tumor given similar clinical and radiological features. Here, we report the case of a patient with a large thigh mass that was ultimately diagnosed as a chronic expanding hematoma. The mass appeared 3 years before the patient visited our clinic and extended from the left flank to the thigh. Diagnostic modalities including computed tomography, magnetic resonance imaging, ultrasonography, and aspiration as well as incisional biopsy suggested that the mass was a malignant tumor such as a liposarcoma or fibrosarcoma; however, this case could not be definitively preoperatively diagnosed as pathologists required the entire mass for diagnostic confirmation. We initially performed a palliative resection rather than a wide radical excision to obtain the whole specimen even though intraoperative findings such as dense adhesion to adjacent tissues and brown liquid material leaking from the mass also suggested the possibility of a malignancy. The final postoperative pathological diagnosis was a benign chronic hematoma.
\end{abstract}

Keywords: Hematoma, Malignancy, Misdiagnosis, Biopsy

\section{Introduction}

Large palpable soft tissue masses can result from a variety of etiologies including benign or malignant tumor, abscess, and hematoma [1]. Hematoma can occur after blunt trauma or surgery, in patients with clotting deficiencies, or spontaneously. In general, hematoma exhibits an acute growth period and resolves spontaneously [2]; however, in some cases, hematoma expands slowly, mimicking a malignant soft tissue tumor. In the 1980s, Reid et al. [3] first defined chronic expanding hematoma as a hematoma which had been developed over 1 month. Okada et al. [1] described three inclusion criteria for chronic expanding hematoma including gradual expansion over a 1-month period, the absence of neoplastic changes on histopathology, and the absence of frequent bleeding. Although some characteristics including invasion of the adjacent tissue or a history of trauma can facilitate a correct diagnosis of chronic expanding hematoma, it is difficult to diagnose chronic expanding hematoma clinically or radiologically [2]. Histopathologically, hematoma consists of a fibrous connective tissue capsule, an inner granulation layer, and old clots [4]. Complete excision is best treatment option for chronic expanding hematoma whereas incomplete resection can result in misdiagnosis and recurrence [2]. To exclude the possibility of soft tissue sarcoma, pathologists often require the whole specimen because chronic hematoma tissue closely resembles the necrotic tissue of a malignant tumor. Here, we report the case of a large soft tissue mass that was preoperatively and intraoperatively misdiagnosed as a soft tissue sarcoma, but ultimately diagnosed

\section{(1) \\ Case Report \\ Received: October 27, 2017 \\ Revised: January 26, 2018 \\ Accepted: January 26, 2018 \\ Corresponding author: \\ Sung Won Jung, M.D. \\ Department of Plastic and Reconstructive Surgery Hallym University Sacred Heart Hospital, Hallym \\ University, 22 Gwanpyeong-ro 170beon-gil, \\ Dongan-gu, Anyang 14068, Korea \\ Tel: +82-31-380-3781 \\ Fax: +82-31-380-5980 \\ E-mail: underwood21@hanmail.net}

This is an Open Access article distributed under the terms of the Creative Commons Attribution Non-Commercial which permits unrestricted non-commercial use, distribution, and reproduction in any medium, provided the original work is properly cited.

(c) 2018 Korean Wound Management Society 
as a chronic hematoma after surgical resection.

\section{Case}

A 58-year-old male patient was referred to our plastic surgery clinic from the department of oncology for treatment of a firm, large, slow-growing mass on his left flank, buttock, and thigh that had developed 3 years prior. The patient had a history of trauma 20 years prior and had previously visited the local clinic, but did not exhibit any other symptoms such as pain or tenderness at the site of the mass. Upon gross examination, a round watermelon-sized cystic mass was observed on the patient's left side spanning from the flank to the thigh (Fig. 1). A blood clotting analysis revealed a slightly prolonged prothrombin time (14.4 s) but no other abnormalities. The medical oncologist first performed diagnostic imaging studies including CT, MRI, and USG. The size of the mass was measured as $16.0 \mathrm{~cm} \times 19.0 \mathrm{~cm} \times 41.0 \mathrm{~cm}$ on MRI (Fig. 2). Radiological reports indicated the possibility of a malignancy such as liposarcoma, fibrosarcoma, or another malignant

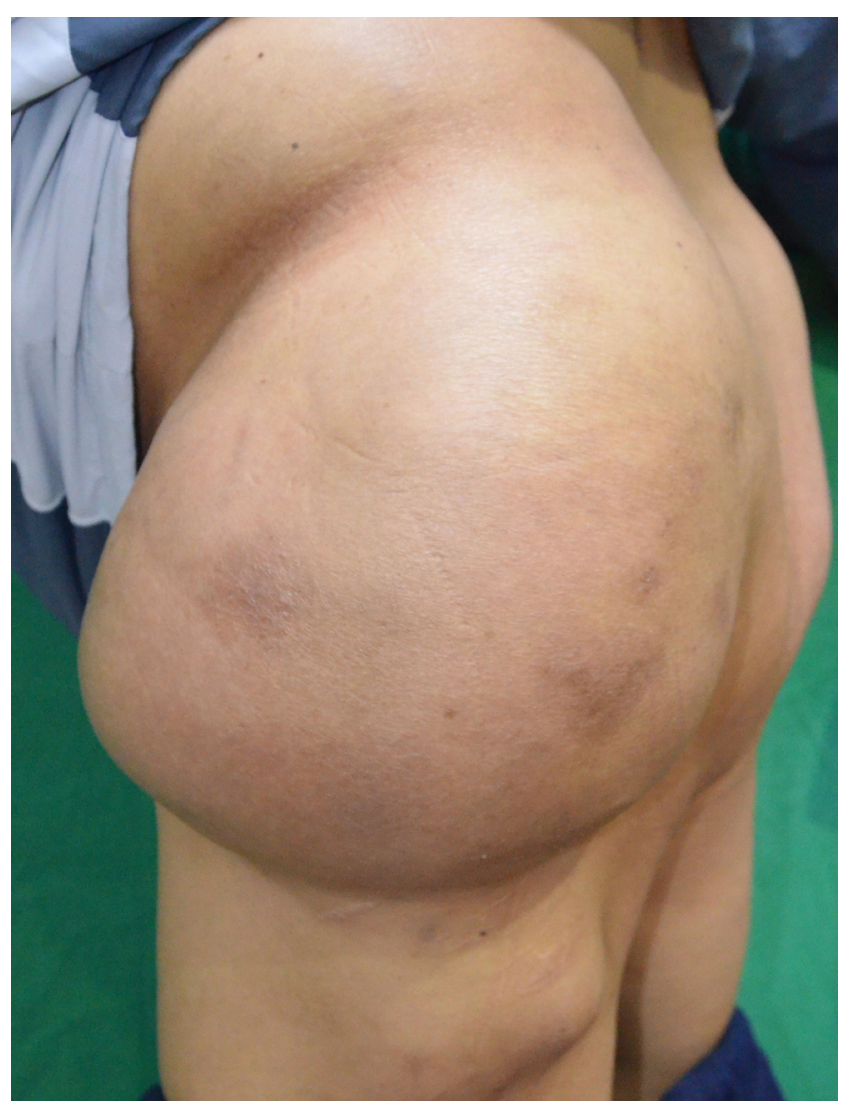

Fig. 1. Gross preoperative view of the huge mass on left buttock and thigh. neurogenic tumor. To exclude potential malignancy, we performed ultrasound-guided and incisional biopsies, but were unable to obtain a definitive diagnosis from histopathology. Accordingly, the whole specimen was required for a diagnosis. We designed a surgical plan and initially organized a team that included general surgeons, plastic surgeons, and orthopedic surgeons because of a chance of peritoneal invasion, exposure of the hip joint, and the size of the defect. We planned an S-shaped long (about $40 \mathrm{~cm}$ ) incision from the left flank to the lateral side of the thigh across the iliac crest. During the procedure, we observed a large mass with dense adhesions to the adjacent tissue and a thick brown liquid that leaked out from the mass. The general and orthopedic surgeons initially speculated that the mass was a malignant tumor, considering that the brown liquid material resembled sarcoma necrosis and that the dense adhesions potentially represented malignant changes. A sample of the mass was removed and immediately sent to the pathologist. From frozen sections, the analysis revealed no tumor cells in the resection margins, but the pathologist was unable to completely exclude the possibility of malignancy (Fig. 3). On this basis, we decided to perform a palliative resection of the entire mass with free tumor margins rather than a radical excision. The incision was closed layer by layer. Histopathology of the entire specimen confirmed that the mass was a chronic he-

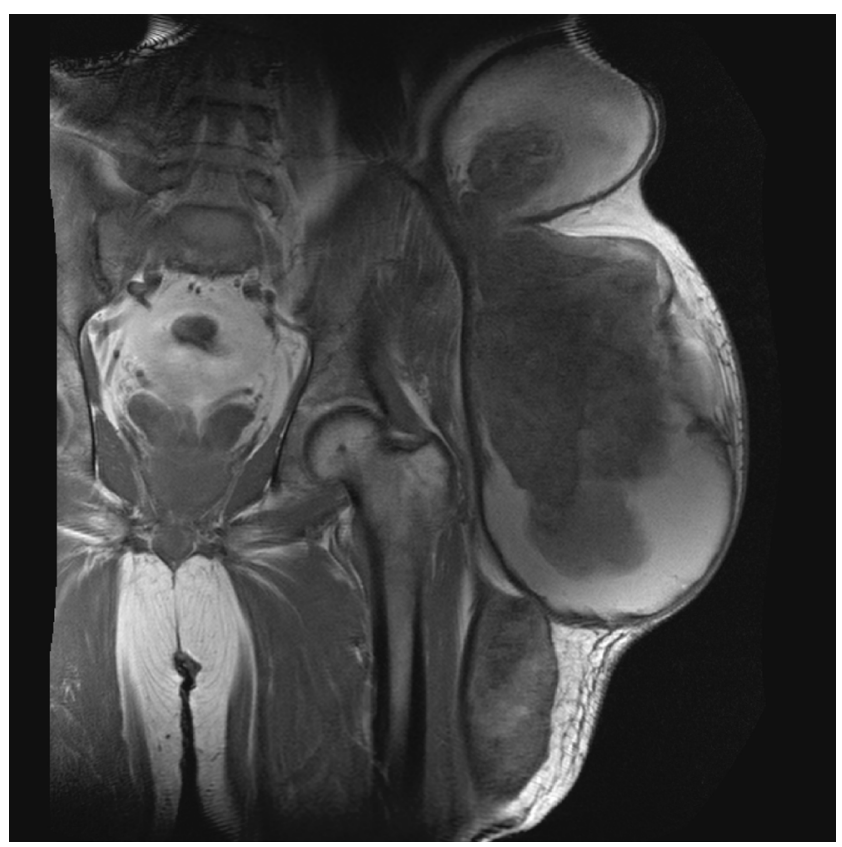

Fig. 2. Preoperative MR imaging of the entire masses (3 lobes). 


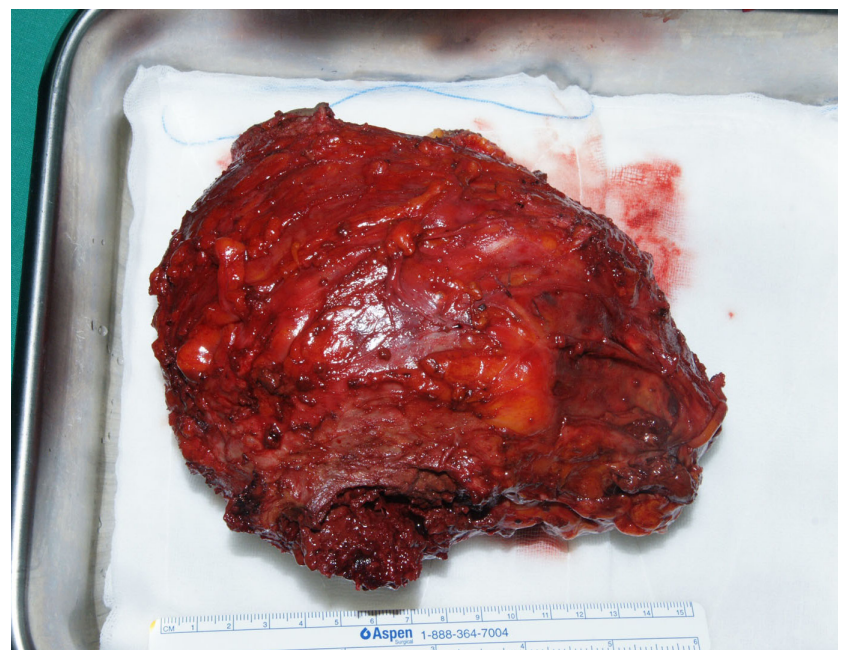

Fig. 3. Intraoperative photograph of the excised specimen.

matoma surrounded by fat tissue. Follow-up at 17 months revealed normal scarring without new presentation of a palpable mass (Fig. 4).

\section{Discussion}

The principal treatment of the primary lesion in a soft tissue sarcoma is radical resection with the aim of preventing local recurrence; however, this can require exposure of the intraperitoneal organs or joints [5]. Therefore, it is difficult to decide between radical excision and palliative resection when the malignancy of a mass is uncertain [6]. Aspiration and incisional biopsy are useful for arriving at a definitive diagnosis in a majority of cases; however, in the present case, histopathological diagnosis was also uncertain and necessitated removal of the whole specimen. We performed a palliative resection rather than a curative resection considering the potential complications associated with wide resection. If the mass had been diagnosed as a malignant tumor after histopathological examination, we would have performed a secondary radical resection with free flap coverage in consideration of potential exposure of the intraperitoneal organs and hip joint.

In conclusion, soft tissue malignancies are particularly difficult to diagnose in cases of possible chronic expanding hematoma, even with the aid of radiological diagnostic tools including CT, MRI, and USG. Pathologic tool like incisional biopsy could also give undefined result. Palliative resection should be considered if the large soft tissue mass is pathologically unconfirmed. Additionally, even when performing

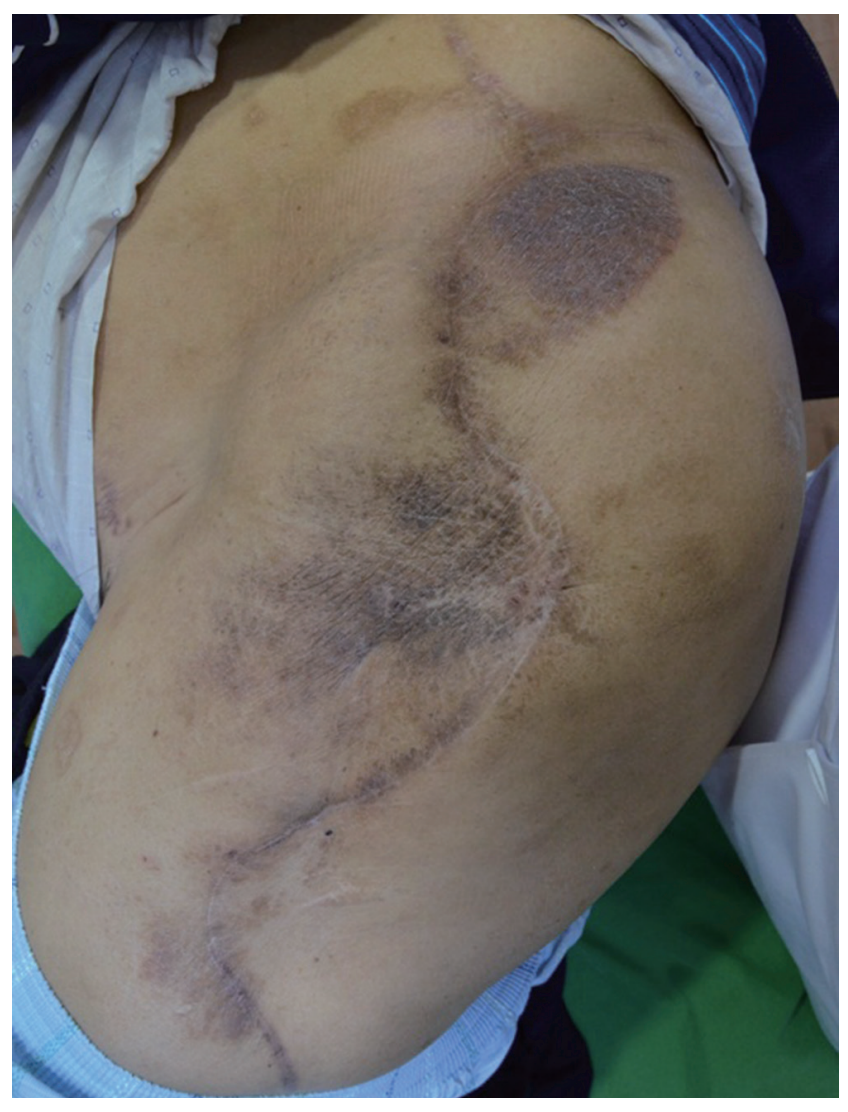

Fig. 4. Gross photograph after 17 months shows no unsightly scar.

radical resection, patients should be preoperatively notified about the possibility of chronic expanding hematoma so that the risks and benefits of a radical resective approach can be adequately considered.

\section{Conflict of interest}

No potential conflict of interest relevant to this article was reported.

\section{References}

1. Okada K, Sugiyama T, Kato H, et al. Chronic expanding hematoma mimicking soft tissue neoplasm. J Clin Oncol 2001; 19:2971-2.

2. Cebesoy O, Tutar E, Arpacioglu O. Spontaneous giant expanding thigh hematoma mimicking soft tissue neoplasm. Joint Bone Spine 2008;75:64-6.

3. Reid JD, Kommareddi S, Lankerani M. Chronic expanding hematomas. A clinicopathologic entity. J Am Med Assoc 1980; 
$\mathrm{Na}$ WG et al.

Diagnostic hardship of soft tissue mass

244:2441-2.

4. Negoro K, Uchida K, Yayama T, et al. Chronic expanding hematoma of the thigh. Joint Bone Spine 2012;79(2):192-4.

5. Goss G, Demetri G. Medical management of unresectable, recurrent low-grade retroperitoneal liposarcoma: integration of cytotoxic and non-cytotoxic therapies into multimodality care. Surg Oncol 2000;9:53-9.

6. Aoki T, Nakata $\mathrm{H}$, Watanabe $\mathrm{H}$, et al. The radiological findings in chronic expanding hematoma. Skeletal Radiol 1999; 28:396-401. 